\title{
BMJ Open Improving neurodevelopmental outcomes in children with congenital heart disease: protocol for a randomised controlled trial of working memory training
}

\author{
Johanna Calderon, ${ }^{1,2}$ David C Bellinger, ${ }^{2}$ Catherine Hartigan, ${ }^{3}$ Alison Lord, ${ }^{3}$ \\ Christian Stopp, ${ }^{3}$ David Wypij, ${ }^{3,4}$ Jane W Newburger ${ }^{3,5}$
}

To cite: Calderon J,

Bellinger DC, Hartigan C, et al. Improving neurodevelopmental outcomes in children with congenital heart disease: protocol for a randomised controlled trial of working memory training. BMJ Open 2019;9:e023304. doi:10.1136/ bmjopen-2018-023304

- Prepublication history and additional material for this paper are available online. To view these files, please visit the journal online (http://dx.doi org/10.1136/bmjopen-2018023304).

Received 30 March 2018 Revised 22 October 2018 Accepted 20 December 2018

D) Check for updates

(c) Author(s) (or their employer(s)) 2019. Re-use permitted under CC BY-NC. No commercial re-use. See rights and permissions. Published by BMJ.

For numbered affiliations see end of article.

\section{Correspondence to}

Dr Johanna Calderon; johanna.calderon@childrens. harvard.edu

\section{ABSTRACT}

Introduction Executive function (EF) impairments are among the most prevalent neurodevelopmental morbidities in youth with congenital heart disease (CHD). To date, no studies have investigated the efficacy of cognitive interventions to improve EF outcomes in children with $\mathrm{CHD}$. Methods and analysis This is a single-centre, singleblinded, two-arm randomised controlled trial to test the efficacy of Cogmed Working Memory Training (Cogmed) versus standard of care in children with CHD after openheart surgery in infancy. Participants will consist of 100 children with CHD aged 7-12 years who underwent openheart surgery before the age of 12 months. Participants are randomly allocated to either an intervention group including training on the home-based Cogmed intervention for a duration of approximately 5 weeks or a control group who receive the standard of care. We will evaluate the efficacy of Cogmed at post-treatment and 3 months after completion of the intervention. Baseline, post-treatment and 3-month follow-up assessments will include specific measures of $\mathrm{EF}$, cognitive and social functioning, and attention deficit hyperactivity disorder (ADHD) symptoms. The primary outcome of this study is the change in standardised mean score on the List Sorting Working Memory test from the National Institutes of Health Toolbox for the Assessment of Neurological and Behavioral Function. Secondary outcomes include measures of social skills, inhibitory control, cognitive flexibility and behavioural EF as well as ADHD symptoms as measured by the Behavior Rating Inventory of Executive Function, Second Edition, and the Conners Third Edition. The efficacy of the intervention will be evaluated by comparing within-subject differences (baseline to posttreatment, baseline to 3-month follow-up) between the two groups using an intention-to-treat analysis.

Ethics and dissemination This study has received Institutional Review Board (IRB) approval from Boston's Children's Hospital IRB (P00022440) and the Human Protection Agency from the US Department of Defense. Trial registration number NCT03023644; Pre-results.

\section{INTRODUCTION}

Congenital heart lesions are among the most common birth defects, ${ }^{12}$ as approximately

\section{Strengths and limitations of this study}

- To our knowledge, this is the first randomised controlled trial (RCT) investigating the efficacy of an executive function intervention in improving outcomes for children with congenital heart disease (CHD).

- The home-based Cogmed Working Memory Training (Cogmed) is among the most widely used evidence-based programmes targeting core executive function skills and will directly address the most frequent neurodevelopmental impairment for children with critical CHD that strongly impacts their ability to succeed in academic and social environments. This intervention is individually adapted to each child's own executive function level, which ensures an optimal level of performance throughout the sessions.

- As a home-based intervention, Cogmed reduces the need for hospital-based treatment visits, potentially reducing the burden for families of children with chronic health conditions such as critical CHD.

- This RCT includes computerised individual measures of neurodevelopment and parent-rating and teacher-rating scales of behavioural and social outcomes as well as collection of patient-specific factors to investigate their potential relationship with response to treatment

- This is a phase II RCT with the goal of providing the first proof of concept that a cognitive intervention can improve outcomes in children with CHD. It is single blinded (investigators are blinded to intervention status and patient characteristics but participants know their treatment group) and has a relatively short duration of follow-up (3 months).

$1 \%$ of infants are born with congenital heart disease (CHD). Of these, more than one-third will present with critical CHD, most broadly defined as forms of CHD requiring surgical or catheter interventions or resulting in death in the first year of life. ${ }^{13}$ Advances in prenatal diagnosis as well as medical and surgical care have reduced mortality rates for all forms 
of CHD. However, evidence of central nervous system damage, including delayed brain maturation in utero and abnormal brain metabolism and microstructure associated with hypoxic-ischaemic injury, has been reported by a wealth of studies of critical CHD. ${ }^{4-7}$ A dramatic increase in the population of survivors of infant heart surgery has been accompanied by the increased recognition of their long-term postoperative morbidities. Neurodevelopmental disabilities, particularly executive function (EF) impairments, are currently the most prevalent long-term morbidity in the population with CHD. ${ }^{4} \mathrm{EF}$ refers to a set of higher order neurocognitive abilities that serve to coordinate and organise actions towards a goal, allowing the individual to adapt to new or complex situations. ${ }^{8}$ Impairments in EF manifest as behavioural dysregulation and attention problems, impaired working memory (ie, the ability to keep information in mind and manipulate it over a short period of time) and problems with organisation and planning abilities. EF is more strongly associated with school readiness than is IQ, predicts both mathematics and reading competence throughout the school years ${ }^{8-10}$ and is strongly associated with social cognition (ie, decoding other people's mental and emotional states and responding to rapid-paced social interactions). ${ }^{9}$

Executive dysfunction can profoundly impact all dimensions of a child's development ${ }^{11-14}$ and is a core feature of attention deficit hyperactivity disorder (ADHD) ${ }^{15}$ and autism spectrum symptoms. ${ }^{16}{ }^{17}$ If untreated, deficits in EF may also predispose individuals to later addiction, ${ }^{18}$ eating disorders and obesity, ${ }^{19}$ and risk-taking behaviours. ${ }^{20}$ These adverse sequelae may carry profound implications for the educational achievement, future employment and quality of life of individuals with CHD. ${ }^{4}$

\section{EF in critical CHD}

Impairments in EF are at the heart of the neurodevelopmental phenotype associated with critical CHD after open-heart surgery. ${ }^{21-34} \mathrm{EF}$ deficits in children with CHD were first reported in school-aged children with dextro-transposition of the great arteries (d-TGAs). ${ }^{25}$ Standardised neuropsychological testing showed that patients with d-TGA had substantial difficulty planning and alternating between tasks, which suggested impairments in cognitive flexibility and working memory as well as deficits in planning and sustained attention. On the Behavior Rating Inventory of Executive Function (BRIEF), parents and teachers of adolescents with CHD reported significant difficulties, particularly with regard to working memory. ${ }^{22}$ Compared with normative values, parents' ratings were worse by $\sim 0.5 \mathrm{SD}$ and those of teachers by $\sim 1 \mathrm{SD}$, suggesting statistically significant and clinically meaningful impairments. More recent findings also reported specific EF impairments in preschool and school-aged children with d-TGA. ${ }^{21} 2324$ In particular, children had important difficulties in behavioural regulation and cognitive control of attention, and they had worse performances on verbal and visual working memory tasks. Consistent findings have been reported by studies that included children with other types of critical CHD such as tetralogy of Fallot ${ }^{28}$ or single ventricle physiology requiring the Fontan operation. ${ }^{29}$ Finally, EF impairments have been associated with worse psychosocial health status and worse quality of life in youth with critical CHD,${ }^{31}$ highlighting the potential impact of longterm executive dysfunction on mental health in CHD. ${ }^{32}$

\section{Working memory intervention for children with CHD}

The American Heart Association (AHA) recommends routine neurodevelopmental screening of all CHD survivors. ${ }^{4} \mathrm{~A}$ burgeoning literature documents the prevalence and importance of impaired EF and ADHD in CHD survivors, ${ }^{21-40}$ and brain imaging studies have provided key information on the underlying disturbances in brain structure and microstructure in patients with CHD. ${ }^{5-7}$ Yet, to date, no trials have been undertaken to test interventions targeting $\mathrm{EF}$ and attention deficits in the $\mathrm{CHD}$ population. $^{34}$

Cogmed Working Memory Training (Cogmed) is the most widely used computerised evidence-based intervention that targets EF, specifically providing intensive structured training of working memory. ${ }^{41-54}$ It has been shown to improve executive performance in several clinical and non-clinical paediatric populations, including children with ADHD, ${ }^{41424647}$ low working memory and low achievement ${ }^{4344}$ and children who were born preterm or extremely low birth weight. ${ }^{53}{ }^{54}$ Unlike hospital-based or laboratory-based interventions, Cogmed can be implemented as a home-based intervention for children. Studies using Cogmed have shown that subjects demonstrate the ability to transfer skills to non-trained tests of working memory as well as to tasks that involve similar processes, including attention, inhibition and non-verbal reasoning. ${ }^{51-53}$ The positive effect of training has been observed on parental ratings of inattention, including the Diagnostic and Statistical Manual of Mental Disorders, Fourth Edition (DSM-IV) Parent Rating Scale, ADHD Rating Scale, Fourth Edition, BRIEF and Conners' Parent Rating Scale. Sustained improvements in behaviour as measured by rating scales have also been observed in ADHD, ${ }^{48}$ brain injury ${ }^{49}$ and non-clinical groups. ${ }^{5152}$

In summary, several randomised controlled trials (RCTs) evaluating the use of Cogmed in healthy children ${ }^{44} 5152$ and in children with various conditions ${ }^{41} 42475354$ have demonstrated that this neurocognitive intervention produces significant generalised and sustained enhancement on measures of $\mathrm{EF}$ and also on everyday life learning and behavioural skills. It is proposed that training working memory using Cogmed is a promising intervention for school-aged children with critical CHD because: (1) it addresses the most frequent neurodevelopmental morbidities that strongly impact the ability to succeed in academic and social environments; (2) it allows for intensive and structured practice of targeted skills, with possible transfer to other neurodevelopmental domains; (3) it is individually adapted to each child's own $\mathrm{EF}$ levels, which ensures an optimal level of performance 
throughout the sessions; (4) it is closely monitored, and various parameters of the child's performance are systematically recorded (eg, correct answers and speed at which tasks are completed); (5) it is child-friendly and rewarding, which facilitates children's compliance; and, finally, but importantly (6) as a home-based intervention, it reduces the need for hospital/clinic-based visits and multiple costs of individual therapy, potentially reducing the burden for families of children with chronic health conditions such as critical CHD.

In this project, we conduct a RCT to provide the first proof of concept that Cogmed intervention improves neurodevelopmental outcomes in children with CHD and that the improvements persist to 3 months. We will enrol children with CHD who underwent infant open-heart surgery as this population corresponds to the highest risk category for developmental disorders and disabilities as per the AHA guidelines (Class I; Level of Evidence A) ${ }^{4}$ We propose to determine immediate and 3-month post-treatment effects on both laboratory-based tests and ecological measures of children's EF, ADHD and social difficulties in everyday life. Our study will also provide insight into factors that are associated with response to treatment, identifying children who may be most likely to benefit from the intervention.

\section{Aims and hypotheses}

Specific aim 1: to evaluate the immediate efficacy of home-based Cogmed intervention for neurodevelopmental outcomes in children with CHD. We hypothesise that children who receive the Cogmed intervention, compared with controls receiving standard of care, will display greater improvement from baseline to post-treatment assessment in EF and social development, and greater reduction in symptoms of ADHD.

Our primary outcome measure will be the change in standardised mean score on the working memory test from the National Institutes of Health Toolbox for the Assessment of Neurological and Behavioral Function (NIH Toolbox) ${ }^{55}$ from baseline to post-treatment. Secondary outcomes include changes in standardised mean scores on tests of cognitive flexibility, attention, inhibitory control and speed of processing from the NIH Toolbox; the Global Executive Composite from the Behavior Rating Inventory of Executive Function, Second Edition (BRIEF-2), ${ }^{56}$ the Global Index and the ADHD Index from the Conners Third Edition (Conners$3)^{57}$ and the Social Responsiveness Scale, Second Edition (SRS-2). ${ }^{58}$

Specific aim 2: to assess the effects of the Cogmed intervention at 3-month follow-up. We predict that significant gains in neurodevelopmental and behavioural outcomes will persist 3 months after cessation of intervention for children who received Cogmed as compared with controls.

The primary and secondary outcomes will be the same as those in specific aim 1, except that the change in scores will be from baseline to 3-month follow-up (ie, approximately 3 months after the last Cogmed session).

Specific Aim 3: to explore cognitive, medical and sociodemographic factors associated with changes in neurodevelopmental and behavioural scores for children who received Cogmed intervention.

\section{METHODS AND ANALYSIS \\ Study design}

This is a single-centre, single-blinded, two-arm RCT to test the efficacy of Cogmed intervention versus standard of care in children with CHD after neonatal and/ or infant open-heart surgery ( $\mathrm{n}=50$ in each group). All eligible subjects undergo a baseline neurodevelopmental assessment and then are randomly assigned to either the home-based Cogmed intervention or to a control group receiving the standard of care for children with CHD. All participants will undergo a post-treatment and a 3-month follow-up assessment. All investigators collecting outcome data are blinded to patients' group assignment (Cogmed intervention versus standard of care) and to medical and surgical histories. Participants and their parents know their group assignment and thus are not blinded. For children assigned to the Cogmed group, post-treatment assessments are performed 1-2 weeks after the end of the intervention (ie, approximately 7-8 weeks after baseline assessment) and follow-up will be performed 3 months after the end of the intervention (ie, approximately 5 months after baseline assessment). For children in the control group, post-treatment and 3-month follow-up assessments are performed approximately $7-8$ weeks and 5 months after baseline assessment, respectively.

\section{Participants and recruitment}

Participants are included if they meet the following criteria: (1) diagnosis of CHD requiring at least one open-heart surgery before 1 year of age; (2) age between 7 years and 12 years at baseline assessment; (3) $\geq 6$ months postcardiac surgery; (4) had received cardiovascular care at Boston Children's Hospital; (5) English or Spanish speaking; and (6) informed consent from parent/guardian as well as assent of the child. Exclusion criteria will be: (1) diagnosed chromosomal anomalies and/or genetic syndromes; (2) severe physical and/or sensory impairments (hearing, visual or psychomotor) that would prevent the use of the computerised program; (3) confirmed diagnosis of an autism spectrum disorder and/or severe developmental or intellectual disorder that would prevent successful completion of the planned study testing; (4) placement in a separate classroom for severe sensory, motor, language or other developmental disability receiving individual support; (5) scheduled to undergo major cardiac interventions in the 6 months following enrolment; and (6) received, receiving or scheduled to receive Cogmed or any other computerised behavioural training programme targeting EF or ADHD. We will not exclude children who underwent multiple 
heart or other surgeries, children with a pre-existing neurological history (eg, epilepsy and stroke) or with a history of a concurrent diagnosis of ADHD (treated or untreated). Rather, we will account for these factors in the data analysis.

Eligible children living in the USA are recruited through patient databases of Boston Children's Hospital Cardiology Clinic and affiliated New England medical centres. Families are invited to participate in the study via a mail packet and follow-up phone call. Flyers and study brochures are displayed in Boston Children's Hospital Cardiology Clinic and affiliated medical centres as well as in some local advocacy parent organisations. Participants are assessed for eligibility and enrolled by a study coordinator and a research nurse. Informed consent and assent from the child are obtained by a study coordinator or a research neuropsychologist before the baseline assessment at the hospital. Parents and children receive monetary compensation for participation in the study. These incentives are given at the second and third visits. Additionally, in order to further facilitate participants' compliance and reduce dropouts, the second and third study assessments may be completed at a child's home.
The study start date (ie, start of active enrolment) was 27 February 2017, and it is anticipated that enrolment will be completed in September 2019.

\section{Patient and public involvement}

Patients, patient/family advocacy groups or the public were not involved in the design, recruitment and conduct of this study. Participants are informed of the burden of the intervention and are given the option to stop at any time point. All eligible patients completing the study will receive an individual report of the results of his or her baseline assessment as well as a general report on study results for the group with CHD when data analyses are completed.

\section{Randomisation and stratification}

All eligible subjects undergo a baseline neurodevelopmental assessment (figure 1) and then are randomly assigned to either the standard home-based Cogmed intervention group or to a control group (standard of care). Allocations are assigned using a computerised system only seen by the research assistant or study coordinator after confirming all eligibility criteria and consent. Subjects are

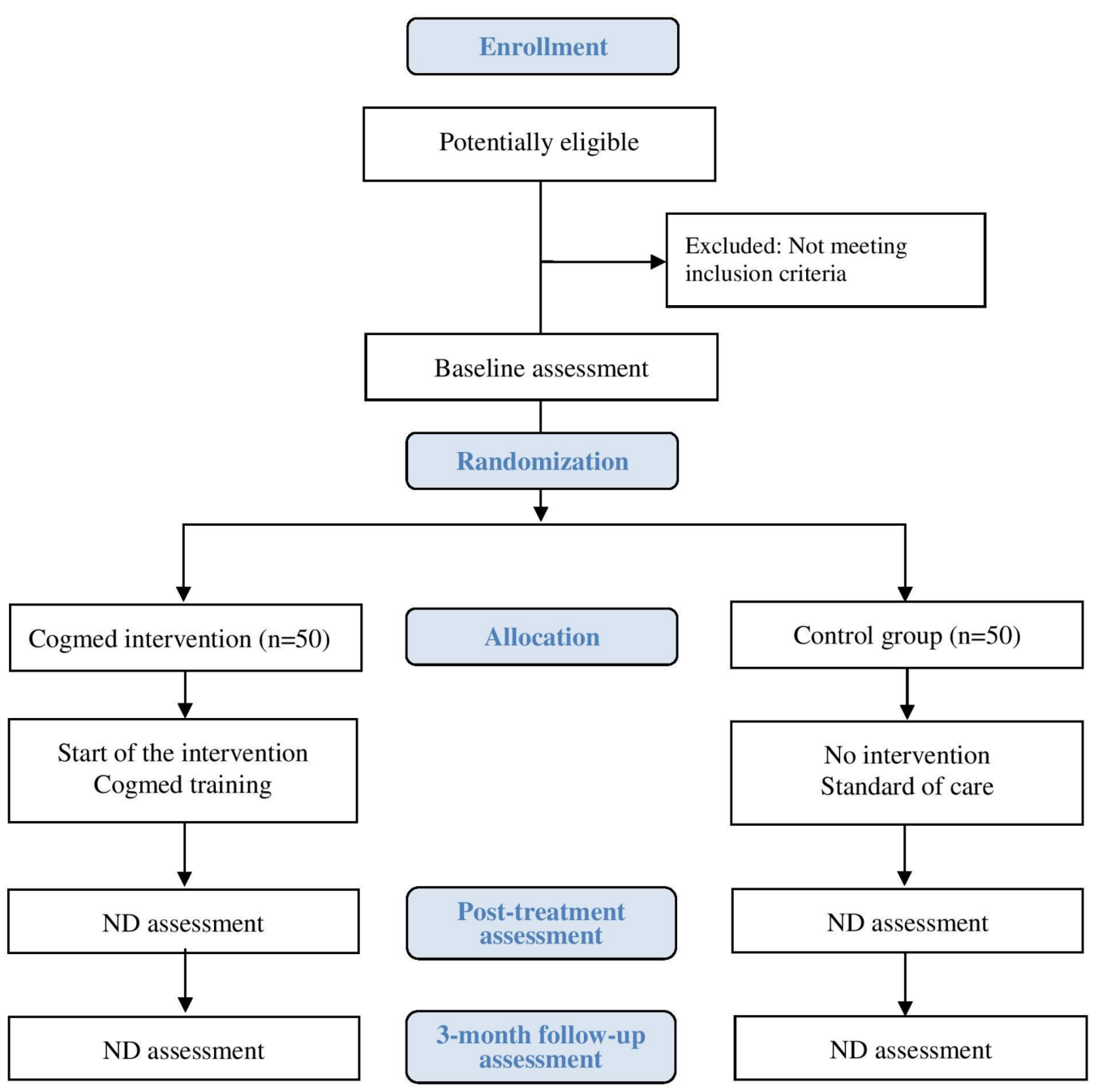

Figure 1 Flow chart of trial design. ND, neurodevelopmental. 
assigned in the order in which they are enrolled into the study. Randomisation is done by computerised permuted blocks design with blocks of varying sizes. Once a subject has been assigned to a group, he or she will remain in the same trial arm for the duration of the study. The randomisation scheme involves two stratification factors: type of CHD (univentricular or biventricular) and baseline level of EF (a score $<85$ or $\geq 85$ on the working memory test from the NIH Toolbox). Figure 1 shows the flow chart of the trial design.

\section{Intervention group: home-based Cogmed Working Memory Training}

Children randomly assigned to receive the Cogmed intervention will complete the standard home-based format of the programme, Cogmed RM, for children aged 7 years and older. The training programme contains 12 childfriendly visual-spatial and verbal working memory tasks (eg, remembering the order in which lamps light up on a $4 \times 4$ grid; recalling a series of numbers of increasing length on the screen). All tasks are adaptive, that is, task complexity levels are automatically adjusted to match each child's working memory capacity, to improve performance and to limit non-compliance to the intervention due to lack of motivation. Tasks become more difficult, on a session-by-session basis, as a child's performance improves. Each training session lasts approximately 40-50 min, and the child is instructed to complete one session per day 5 days each week for 5 weeks, for a total of 25 sessions. The programme yields individual sessionby-session and task-by-task training results, including the children's responses, time spent on each task and evolution curves. Cogmed is not Food and Drug Administration (FDA)-regulated. Based on our specific aims, Cogmed is considered a non-significant risk device.

Study tablets (ie, iPads) are provided to families randomised to the Cogmed group in order to standardise the method of delivery. Families receive a link for downloading a web-based software program to the tablet. The program is installed on the tablet by a study coordinator who explains how the training program works and how to $\log$ into the system and complete training. The training session and installation of the program are completed after baseline assessment and randomisation. Parents and children will be actively involved, and during the installation session, children will complete several practice trials under the supervision of the study coordinator. The 25 sessions will be completed by the child, supervised by a parent. For the first five sessions, the child trains on the same set of games; on the sixth session and every fifth session thereafter, a new task is introduced and replaces one of the initial tasks. At the end of each session, the child can play an age-appropriate tablet game as a reward. After each session, a parent will upload the results to a secure website. Families are contacted weekly to check program function and discuss concerns. Compliance is automatically registered by the computerised program and is defined as completing at least 20 sessions, the criterion by which children are categorised as compliant or non-compliant to treatment. ${ }^{41} 42$

To implement this intervention, each investigator and study coordinator involved in coaching is certified as a 'Cogmed Coach'. The Cogmed Coaches will monitor children's performance on mycogmed.com secured website every week during the intervention to permit continuous evaluation of treatment compliance and fidelity. The Cogmed coach specifically monitors performance of each child and contacts the parents and the child by phone on a weekly basis to discuss progression and any issues arising during the training week. A designated Cogmed coach will be available during the trial to respond to any questions or help with any difficulties during the training. Families and children are encouraged to continue the training for at least 20 sessions. If parents or children struggle with some aspects of the intervention such as the time commitment or a lack of motivation to persist with the training, the Cogmed coach discusses alternative options for accommodating each individual child's needs (ie, rewards systems available, best time of the day to practice, number of breaks necessary during each session and so on) We provide weekly feedback sessions and close monitoring in order to discourage dropouts and increase compliance with the intervention. Parents are asked to complete a training evaluation scale following completion of Cogmed; this scale is an integrated component of Cogmed that gathers information regarding the child's motivation and attention during the training as well as parents' feedback. As soon as a child finishes the intervention, a blinded post-treatment assessment will be scheduled to occur within the following weeks.

\section{Control group: standard of care}

Children randomly assigned to the control group will receive the standard of care recommended for patients with critical CHD. This includes cardiac surveillance and, if needed, neurodevelopmental counselling and screening at Boston Children's Hospital Cardiac Neurodevelopmental Program. Once enrolled in the study, a child in the control group will not receive Cogmed intervention or any other cognitive intervention that targets EFs or ADHD symptoms until after the 3-month follow-up assessment is completed, that is, 5-6 months after initial enrolment. Like children assigned to the intervention group, children in the control group can continue treatments that are already in place for other neurodevelopmental disabilities (eg, speech therapy and occupational services). For children in the control group, post-treatment and 3-month follow-up assessments will be performed 6-7 weeks and 4-5 months after baseline assessment, respectively. After the study is completed, children in the control group will be offered the possibility of completing the Cogmed intervention at no cost.

\section{Primary outcome measure}

The NIH Toolbox ${ }^{55}$ is a set of computerised assessments designed to measure outcomes in longitudinal or 
intervention trials. This battery is particularly appropriate for our study because it is presented in a computerised child-friendly version, paralleling that of the Cogmed intervention. The List Sorting Working Memory Test from the NIH Toolbox is the primary outcome of the trial. This standardised measure assesses the ability to process information across a series of modalities (visual-spatial and verbal), to hold this information in a short-term buffer and to actively manipulate it mentally. It is considered an excellent composite indicator of children's EF skills, as it requires the simultaneous implementation of control of attention and working memory abilities on tasks of increasing complexity. Mean scores are automatically computed and are compared with a standardisation sample of US children of the same age. Scores are normally distributed (mean $=100, \mathrm{SD}=15$ ) in the standardisation sample. The construct validity of the NIH Toolbox working memory tasks is 0.58 for convergent validity and 0.30 for divergent validity. This test has a test-retest reliability of 0.89 (95\% CI 0.85 to 0.92$)$.

\section{Secondary outcome measures}

$\mathrm{NIH}$ toolbox cognition battery

We include tests that measure cognitive flexibility, attention and inhibitory control, episodic memory, language and processing speed. Mean scores on the following tests will be our secondary outcomes: (1) Flanker Inhibitory Control and Attention Test, which measures a child's ability to control automatic response tendencies that may interfere with achieving a goal; (2) Dimensional Change Card Sort Test, which assesses a child's capacity to switch among multiple aspects of a task; (3) Picture Sequence Memory Test, which measures a child's ability to remember the sequence of pictures shown on the screen; (4) Picture Vocabulary Test and Oral Reading Recognition, which assess receptive vocabulary and reading decoding skills; and (5) Pattern Comparison Processing Speed Test, which assesses the amount of time it takes a child to process a set amount of information. All scores are standardised and normally distributed (mean=100, $\mathrm{SD}=15$ ) in the standardisation sample. The test-retest reliability of these tests varies between 0.82 and $0.96 .^{55}$

\section{Behaviour Rating Inventory of Executive Function, Second Edition}

The BRIEF-2 is a standardised questionnaire that measures children's executive functioning in everyday life. It includes nine scales: inhibit, self-monitor, shift, emotional control, initiate, working memory, plan/organise, task-monitor and organisation of materials. Parent and teacher versions of the BRIEF-2 will be included. We will analyse the General Executive Composite $\mathrm{T}$ score (mean=50, SD=10 for the standardisation sample) for each version (Parent and Teacher), which integrates a child's scores on all of the clinical scales. The composite indices of the BRIEF-2 have high internal consistency (0.94-0.98 in the normative sample) and high test-retest reliability (0.84-0.88 for parents over a 2-week interval; $0.90-0.92$ for teachers over a 3.5 -week interval) ${ }^{56}$
Conners, Third Edition

The Conners-3 is a questionnaire that assesses ADHD-related behaviours in children aged 3-17 years. We will analyse mean $\mathrm{T}$ scores (mean $=50, \mathrm{SD}=10$ in the standardisation sample) for the ADHD Inattentive and the ADHD Hyperactive-Impulsive DSM-5 Symptom Scales as well as the ADHD Index for both the Parent and Teacher versions. For children aged 6-11 years, the Cronbach's alpha coefficients for scores on the scales range from 0.87 to 0.95 for both parent and teacher ratings, indicating satisfactory internal consistency. Test-retest reliability for the scales ranges from 0.67 to 0.72 for parents and 0.47 0.80 for teachers. ${ }^{57}$

\section{Social Responsiveness Scale, Second Edition}

The SRS-2 questionnaire evaluates autism spectrum symptoms, including those relating to social awareness, social cognition, communication, social motivation and autistic traits, in individuals older than 2.5 years. We will analyse $\mathrm{T}$ scores (mean $=50, \mathrm{SD}=10$ in the standardisation sample) from both versions (Parent and Teacher). Ratings show good internal consistency and inter-rater reliability. ${ }^{58}$

The schedule of neurodevelopmental data collection is presented in table 1 .

\section{Covariate measures}

We will investigate cognitive, medical and sociodemographic patient-specific factors as predictors of response to the intervention, at both post-treatment and 3-month follow-up assessments. The following variables will be investigated: baseline Full-Scale IQ scores and all subscales on the Wechsler Intelligence Scale for Children, Fifth Edition, ${ }^{59}$ and perinatal medical history, including birth weight, gestational age, type of CHD, history of neurological abnormalities, number of open-heart surgeries, intensive care unit length of stay and total number of hospitalisations.

\section{Data management and safety monitoring}

Overall integration of the statistics, data management and administrative functions of this trial occur in the Department of Cardiology's Research Support and Statistics Core (RSSC) led by Drs Jane Newburger and David Wypij. The RSSC provides the infrastructure necessary to facilitate the conduct of this clinical trial including biostatistical analysis, computerised data entry, data base programming and development, data management, quality control, assistance with manuscript preparation and administrative functions. The RSSC provides a centralised resource for maintaining database. Study documents are being stored in individual subject folders, each folder containing a tracking page. All study materials are stored in a locked file cabinet accessible only to authorised study staff. All study data are recorded on case report forms and entered into a Research Electronic Data Capture database.

A Data and Safety Monitoring Board (DSMB) is composed of expert members in cardiology, 
Table 1 Schedule of neurodevelopmental assessment data collection

\begin{tabular}{|c|c|c|c|c|}
\hline Assessment & Informant & Baseline & Post-treatment* & Follow-upt \\
\hline \multicolumn{5}{|l|}{ Primary outcome } \\
\hline NIH Toolbox List Sorting Working Memory Test & Child & $x$ & $\mathrm{X}$ & $\mathrm{X}$ \\
\hline NIH Toolbox Cognition Battery & Child & $\mathrm{X}$ & $\mathrm{X}$ & $\mathrm{x}$ \\
\hline $\begin{array}{l}\text { Behavior Rating Inventory of Executive Function, } \\
\text { Second Edition }\end{array}$ & Parent & $x$ & $x$ & $\mathrm{x}$ \\
\hline \multirow[t]{2}{*}{ Conners, Third Edition } & Parent & $x$ & $\mathrm{x}$ & $\mathrm{x}$ \\
\hline & Teacher & $\mathrm{X}$ & $\mathrm{X}$ & $\mathrm{x}$ \\
\hline \multirow[t]{2}{*}{ Social Responsiveness Scale, Second Edition } & Parent & $x$ & $x$ & $x$ \\
\hline & Teacher & $X$ & $\mathrm{X}$ & $\mathrm{X}$ \\
\hline
\end{tabular}

*Post-treatment (one to 2 weeks after cessation of intervention and/or 6-7 weeks after baseline).

†Three-month follow-up (3 months after completion of the intervention and/or 4-5 months after baseline).

neuropsychology and biostatistics. Members will be independent of the study investigators and their Departments at Boston Children's Hospital as well as from the sponsors of this study. The function of the DSMB will be to advise the funding sources, Boston Children's Hospital and the study investigators on: (1) final study designs and protocols prior to the beginning of data collection, (2) problems encountered protocol implementation, (3) frequency of occurrence of adverse events and their relation to study protocols, (4) withdrawals and losses to follow-up, (5) data interpretation and ethical issues and (6) recommendations arising from the study. The DSMB chair will receive reports of any serious events that occur in the conduct of the study. This trial has been considered as a non-significant risk device study and reviewed accordingly by the Boston Children's Hospital Institutional Review Board and the Human Research Protection Office, US Department of Defense.

A complete description of this trial's data management plan, safety monitoring board and risk/benefits assessment is presented in online appendix 1 .

\section{Sample size and power considerations}

Our specific aims are to determine whether there are significant differences between the intervention and control groups in the change in scores on the List Sorting Working Memory Test between measurements at baseline and post-treatment (specific aim 1) and between baseline and 3-month follow-up (specific aim 2). Although this test has a good test-retest reliability of $\rho=0.87$, to be conservative, we will assume a value of $\rho=0.70$ between baseline and post-treatment and between baseline and 3-month follow-up on the same subject. Given a sample size of 100 subjects, $\rho=0.70$ for within-subject correlations, and a two-sided type I error rate of $5 \%$, we have $81.4 \%$ power to detect a mean difference of $0.5 \mathrm{SD}$ between treatment groups, with a conservative $20 \%$ attrition rate (hence, analysing a minimum of 80 subjects) in our primary outcome measure. This corresponds to a mean difference of 7.5 units, given an expected SD of 15 for the List Sorting Working Memory Test of the NIH Toolbox.

Among children who receive the Cogmed intervention, we also seek to assess associations of cognitive, sociodemographic and medical factors with changes in the scores for our primary outcome measure (specific aim 3). Given a sample size of 50 children in the Cogmed group and a two-sided type I error rate of $5 \%$, we have $79.9 \%$ power to detect a correlation of 0.43 (or $R^{2}=0.43^{2}=0.185$ from a linear regression) between a patient-specific factor and the primary outcome variable even with a conservative $20 \%$ attrition rate (analysing a minimum of 40 subjects).

\section{Data analysis plan}

For specific aims 1 and 2, the efficacy of the intervention will be evaluated by comparing within-subject differences (baseline to post-treatment, baseline to 3-month follow-up and, in secondary analyses, post-treatment to 3-month follow-up) across treatment groups using an intention-totreat analysis.

Descriptive statistics will be calculated, including means, SD, medians and IQRs for continuous variables and frequency counts and percentages for categorical variables. The primary outcome measure, the List Sorting Working Memory Test of the NIH Toolbox and most other study outcomes are continuous variables. T-tests and linear regression will be used to assess differences between the intervention and control groups for continuous outcomes (ie, differences in means, 95\% CI). Proportions and logistic regression will be used to examine group differences in binary outcomes (ie, $\chi^{2}$ tests, ORs and 95\% CI). We expect that randomisation will produce balance between treatment groups in terms of demographic and baseline factors, but we will use regression methods to adjust for any factors that may be unbalanced. All analyses will be accompanied by graphical exploration of the data to identify outlying 
and influential observations. Data transformations and non-parametric methods (eg, Wilcoxon rank-sum tests) will be used as appropriate when parametric assumptions are violated. Primary analyses of treatment group differences will focus on complete cases. In secondary analyses, we will assume no change over time for subjects who do not return for their post-treatment assessment (ie, last value carried forward approach), but we will also carry out other sensitivity analyses to assess the strength of our findings based on other missing data assumptions.

For specific aim 3, we will explore the associations between patient factors and within-subject differences (baseline to post-treatment, baseline to 3-month follow-up) using correlation and linear regression methods, including consideration of possible confounding or effect modification. Specific attention will be given to certain patient-specific risk factors including age at first heart surgery (neonatal vs non-neonatal), number of surgeries and neurological complications. Because we will be conducting multiple analyses with several predictors and primary and secondary outcomes in an exploratory fashion, we will interpret results cautiously, based on significance levels $(p<0.05$, two tailed) and on the magnitude of differences, correlations or regression effects. As appropriate, we will also consider the use of other statistical methods, such as generalised additive models, partial and sparse partial least squares and family-wise error rates, in our approach. Analyses will be conducted primarily using SAS V.9.4, Stata V.15.1, SPSS V.25 and R V.3.5.2.

\section{ETHICS AND DISSEMINATION}

Protocol modifications and amendments will be submitted to the ethical committees for approval. Amendments to the study protocol will be added to publications reporting the study outcomes. This trial has been registered with the American Clinical Trials Registry. Prior to entering into the trial, all parents or legal guardians and children will give written informed consent or assent to participate. Online appendix 2 presents the study consent form. All information will follow IRB and Human Protection guidelines for confidentiality and data protection. The study results will be disseminated through publications in scientific journals, presentations at scientific conferences and directly to the families who participated in the study. Coinvestigators will be coauthors of the publications describing trial outcomes, without the use of professional writers. Data will be provided on request.

\section{Trial progress}

The trial is currently in the active recruitment phase (first baseline assessment February 2017). This is Protocol V.4, 10 July 2018. Substantial protocol amendments will be communicated to investigators via email and to other parties as required. All changes are submitted to Boston
Children's Hospital's IRB, to the Sponsor of this trial (US Department of Defense) and updated in clinicaltrials.gov.

\section{Discussion}

This article presents the background and design of an RCT investigating the efficacy of a 5-week working memory intervention for children with CHD who underwent open-heart surgery in infancy. This is the first study to investigate the effects of a neurocognitive intervention targeting EF in school-aged children with CHD. We will evaluate children's cognitive and social outcomes including autism spectrum and ADHD symptoms. Furthermore, the results from this trial will provide information on potential patient-specific factors associated with response to the intervention. As a first clinical trial, we will test the efficacy of the intervention at 3 months after the cessation of training. If the intervention is proven effective at this time, longer term effects should be investigated (eg, at 6-month or 12-month postintervention). Assessment of longer term effects of working memory training will provide key information about the cost-efficacy of Cogmed in patients with CHD, the likelihood that lasting benefits generalise to other areas of development and the duration of its benefits.

Executive dysfunction may have cascading adverse effects on a myriad of domains ranging from specific neurocognitive abilities to school achievement, social adaptation and, ultimately, quality of life. Timely prevention and treatment of these issues is a priority in the care of patients with CHD. If proven effective, this type of neurocognitive intervention could be implemented in a clinical outpatient practice for patients at increased neurodevelopmental risk.

\section{Author affiliations}

${ }^{1}$ Boston Children's Hospital, Department of Psychiatry, Boston, Massachusetts, USA ${ }^{2}$ Department of Psychiatry, Harvard Medical School, Boston, Massachusetts, USA ${ }^{3}$ Department of Cardiology, Boston Children's Hospital, Boston, Massachusetts, USA ${ }^{4}$ Department of Biostatistics, Harvard T.H. Chan School of Public Health, Boston, Massachusetts, USA

${ }^{5}$ Department of Pediatrics, Harvard Medical School, Boston, Massachusetts, USA

Contributors JC, DCB and JWN are the primary investigators and, together with DW, designed and established this research study. JC and JWN were responsible for ethics applications and reporting. JC, DCB, CH and AL are responsible for data collection and implementation of the intervention. JC will take lead roles on preparation for publication of the clinical outcomes of the study. JWN, DCB, DW, CS, $\mathrm{CH}$ and $\mathrm{AL}$ will contribute to the preparation of publications within their respective fields of expertise. DW and CS will take on a lead role of the statistical analysis for the study. JC drafted the final version of this manuscript. All authors critically reviewed and approved the final version. All data from this study will be submitted to peer-review journals and for presentation at national and international scientific conferences.

Funding This research was funded by the US Department of Defense, Clinical Trials awards (grant number W81XWH-16-1-0741).

\section{Competing interests None declared.}

Patient consent for publication Not required.

Ethics approval Full ethical approval for this study has been obtained by the Boston Children's Hospital's Institutional Review Board (IRB) (IRB number P00022440) and has also been reviewed and approved by the Human Protection Agency from the US Department of Defense. All parent/guardians and children will give written informed consent or assent to participate prior to entering into the trial. 
Provenance and peer review Not commissioned; externally peer reviewed.

Open access This is an open access article distributed in accordance with the Creative Commons Attribution Non Commercial (CC BY-NC 4.0) license, which permits others to distribute, remix, adapt, build upon this work non-commercially, and license their derivative works on different terms, provided the original work is properly cited, appropriate credit is given, any changes made indicated, and the use is non-commercial. See: http://creativecommons.org/licenses/by-nc/4.0/.

\section{REFERENCES}

1. Fyler DC. Report of the New England Regional Infant Cardiac Program. Pediatrics 1980;65:S375-61.

2. Loffredo CA. Epidemiology of cardiovascular malformations: prevalence and risk factors. Am J Med Genet 2000;97:319-25.

3. Ferry PC. Neurologic sequelae of cardiac surgery in children. Am J Dis Child 1987;141:309-12.

4. Marino BS, Lipkin PH, Newburger JW, et al. Neurodevelopmental outcomes in children with congenital heart disease: evaluation and management: a scientific statement from the American Heart Association. Circulation 2012;126:1143-72.

5. Mahle WT, Tavani F, Zimmerman RA, et al. An MRI study of neurological injury before and after congenital heart surgery. Circulation 2002;106(Suppl 1):109-14.

6. Miller SP, McQuillen PS, Hamrick S, et al. Abnormal brain development in newborns with congenital heart disease. $N$ Engl $J$ Med 2007;357:1928-38

7. Owen M, Shevell M, Majnemer A, et al. Abnormal brain structure and function in newborns with complex congenital heart defects before open heart surgery: a review of the evidence. $J$ Child Neurol 2011;26:743-55

8. Diamond A. Executive functions. Annu Rev Psychol 2013;64:135-68.

9. Blair C, Razza RP. Relating effortful control, executive function, and false belief understanding to emerging math and literacy ability in kindergarten. Child Dev 2007;78:647-63.

10. Diamond A, Barnett WS, Thomas J, et al. Preschool program improves cognitive control. Science 2007;318:1387-8.

11. Bull R, Scerif G. Executive functioning as a predictor of children's mathematics ability: inhibition, switching, and working memory. Dev Neuropsychol 2001:19:273-93.

12. Biederman J, Monuteaux MC, Doyle AE, et al. Impact of executive function deficits and attention-deficit/hyperactivity disorder (ADHD) on academic outcomes in children. J Consult Clin Psychol 2004;72:757-66.

13. Espy KA, McDiarmid MM, Cwik MF, et al. The contribution of executive functions to emergent mathematic skills in preschool children. Dev Neuropsychol 2004;26:465-86.

14. Diamond A. Normal development of prefrontal cortex from birth to young adulthood: cognitive functions, anatomy, and biochemistry. In: Stuss D, Knight RT, eds. Principles of Frontal Lobe Function. New Yord, NY: Oxford University Press, 2002:466-503.

15. Barkley RA. Distinguishing sluggish cognitive tempo from ADHD in children and adolescents: executive functioning, impairment, and comorbidity. J Clin Child Adolesc Psychol 2013;42:161-73.

16. Lai M-C, Lombardo MV, Baron-Cohen S. Autism. The Lancet 2014;383:896-910.

17. Pellicano E. Individual differences in executive function and central coherence predict developmental changes in theory of mind in autism. Dev Psychol 2010;46:530-44.

18. Baler RD, Volkow ND. Drug addiction: the neurobiology of disrupted self-control. Trends Mol Med 2006;12:559-66.

19. Miller AL, Lee HJ, Lumeng JC. Obesity-associated biomarkers and executive function in children. Pediatr Res 2015;77(1-2):143-7.

20. Levy S, Katusic SK, Colligan RC, et al. Childhood ADHD and risk for substance dependence in adulthood: a longitudinal, populationbased study. PLoS One 2014;9:e105640.

21. Calderon J, Jambaqué I, Bonnet $\mathrm{D}$, et al. Executive functions development in 5- to 7-year-old children with transposition of the great arteries: a longitudinal study. Dev Neuropsychol 2014;39:365-84.

22. Bellinger DC, Wypij D, Rivkin MJ, et al. Adolescents with d-transposition of the great arteries corrected with the arterial switch procedure: neuropsychological assessment and structural brain imaging. Circulation 2011;124:1361-9.

23. Calderon J, Bonnet $\mathrm{D}$, Courtin $\mathrm{C}$, et al. Executive function and theory of mind in school-aged children after neonatal corrective cardiac surgery for transposition of the great arteries. Dev Med Child Neurol 2010:52:1139-44.
24. Calderon J, Angeard N, Moutier S, et al. Impact of prenatal diagnosis on neurocognitive outcomes in children with transposition of the great arteries. J Pediatr 2012;161:94-8.

25. Bellinger DC, Wypij D, duPlessis AJ, et al. Neurodevelopmental status at eight years in children with dextro-transposition of the great arteries: the Boston Circulatory Arrest Trial. J Thorac Cardiovasc Surg 2003;126:1385-96.

26. Bellinger DC, Rivkin MJ, DeMaso D, et al. Adolescents with tetralogy of Fallot: neuropsychological assessment and structural brain imaging. Cardiol Young 2015;25:338-47.

27. Miatton M, De Wolf D, François K, et al. Neuropsychological performance in school-aged children with surgically corrected congenital heart disease. J Pediatr 2007;151:73-8.

28. Hövels-Gürich HH, Konrad K, Skorzenski D, et al. Attentional dysfunction in children after corrective cardiac surgery in infancy. Ann Thorac Surg 2007;83:1425-30.

29. Gaynor JW, Gerdes M, Nord AS, et al. Is cardiac diagnosis a predictor of neurodevelopmental outcome after cardiac surgery in infancy? J Thorac Cardiovasc Surg 2010;140:1230-7.

30. Cassidy AR, White MT, DeMaso DR, et al. Executive function in children and adolescents with critical cyanotic congenital heart disease. J Int Neuropsychol Soc 2015;21:34-49.

31. Neal AE, Stopp C, Wypij D, et al. Predictors of health-related quality of life in adolescents with tetralogy of Fallot. $J$ Pediatr 2015:166:132-8.

32. Calderon J, Bellinger DC. Executive function deficits in congenital heart disease: why is intervention important? Cardiol Young 2015;25:1238-46.

33. Shillingford AJ, Glanzman MM, Ittenbach RF, et al. Inattention, hyperactivity, and school performance in a population of schoolage children with complex congenital heart disease. Pediatrics 2008;121:e759-67.

34. Hansen E, Poole TA, Nguyen V, et al. Prevalence of ADHD symptoms in patients with congenital heart disease. Pediatr Int 2012;54:838-43.

35. DeMaso DR, Labella M, Taylor GA, et al. Psychiatric disorders and function in adolescents with d-transposition of the great arteries. $J$ Pediatr 2014;165:760-6.

36. Newburger JW, Sleeper LA, Bellinger DC, et al. Early developmental outcome in children with hypoplastic left heart syndrome and related anomalies: the single ventricle reconstruction trial. Circulation 2012:125:2081-91.

37. Yamada DC, Porter AA, Conway JL, et al. Early repair of congenital heart disease associated with increased rate of attention deficit hyperactivity disorder symptoms. Can J Cardiol 2013;29:1623-8.

38. Sistino JJ, Atz AM, Simpson KN, et al. The prevalence of attentiondeficit/hyperactivity disorder following neonatal aortic arch repair. Cardiol Young 2015;25:663-9.

39. von Rhein M, Buchmann A, Hagmann C, et al. Brain volumes predict neurodevelopment in adolescents after surgery for congenital heart disease. Brain 2014;137(Pt 1):268-76.

40. Schaefer C, von Rhein M, Knirsch W, et al. Neurodevelopmental outcome, psychological adjustment, and quality of life in adolescents with congenital heart disease. Dev Med Child Neurol 2013:55:1143-9.

41. Klingberg T, Fernell E, Olesen PJ, et al. Computerized training of working memory in children with ADHD-a randomized, controlled trial. J Am Acad Child Adolesc Psychiatry 2005;44:177-86.

42. Klingberg $\mathrm{T}$, Forssberg $\mathrm{H}$, Westerberg $\mathrm{H}$. Training of working memory in children with ADHD. J Clin Exp Neuropsychol 2002;24:781-91.

43. Holmes J, Gathercole SE, Dunning DL. Adaptive training leads to sustained enhancement of poor working memory in children. Dev Sci 2009:12:F9-F15.

44. Holmes J, Gathercole SE. Taking working memory training from the laboratory into schools. Educ Psychol 2014;34:440-50.

45. Westerberg $\mathrm{H}$, Klingberg $\mathrm{T}$. Changes in cortical activity after training of working memory-a single-subject analysis. Physiol Behav 2007;92(1-2):186-92.

46. Brehmer $\mathrm{Y}$, Westerberg $\mathrm{H}$, Bäckman L. Working-memory training in younger and older adults: training gains, transfer, and maintenance. Front Hum Neurosci 2012;6:63.

47. Beck SJ, Hanson CA, Puffenberger SS, et al. A controlled trial of working memory training for children and adolescents with ADHD. $J$ Clin Child Adolesc Psychol 2010;39:825-36.

48. Gropper RJ, Gotlieb H, Kronitz R, et al. Working memory training in college students with ADHD or LD. J Atten Disord 2014;18:331-45.

49. Johansson B, Tornmalm M. Working memory training for patients with acquired brain injury: effects in daily life. Scand $J$ Occup Ther 2012;19:176-83.

50. Lundqvist A, Grundström K, Samuelsson K, et al. Computerized training of working memory in a group of patients suffering from acquired brain injury. Brain Inj 2010;24:1173-83. 
51. Bergman Nutley S, Söderqvist S, Bryde S, et al. Gains in fluid intelligence after training non-verbal reasoning in 4-year-old children: a controlled, randomized study. Dev Sci 2011:14:591-601.

52. Thorell LB, Lindqvist S, Bergman Nutley S, et al. Training and transfer effects of executive functions in preschool children. Dev Sci 2009;12:106-13.

53. Grunewaldt KH, Løhaugen GC, Austeng D, et al. Working memory training improves cognitive function in VLBW preschoolers. Pediatrics 2013;131:e747-54.

54. Løhaugen GC, Antonsen I, Håberg A, et al. Computerized working memory training improves function in adolescents born at extremely low birth weight. J Pediatr 2011;158:555-61.
55. Zelazo PD, Anderson JE, Richler J, et al. II. NIH Toolbox Cognition Battery (CB): measuring executive function and attention. Monogr Soc Res Child Dev 2013;78:16-33.

56. Baron IS. Behavior rating inventory of executive function. Child Neuropsychol 2000;6:235-8.

57. Conners CK. Manual for the Conners Rating Scales - Revised. North Tonawanda, NY: MHS Assessments, 1997.

58. Constantino JN, Davis SA, Todd RD, et al. Validation of a brief quantitative measure of autistic traits: comparison of the social responsiveness scale with the autism diagnostic interview-revised. $J$ Autism Dev Disord 2003;33:427-33.

59. Wechsler D. Wechlser Intelligence Scale for Children. 5th edn: Psychological Corporation, Inc, 2014. 